\title{
A potential mechanism of action of dietary anthocyanins: modulation of endothelial superoxide production by phenolic acid metabolites
}

\author{
G. M. Woodward, A. Cassidy and C. D. Kay \\ Department of Nutrition, Norwich Medical School, University of East Anglia, Norwich, NR4 7TJ, UK
}

It has long been thought that diets rich in anthocyanins confer protection against the oxidative progression of CVD. Although inflammatory cell superoxide production is believed to be a major contributor to oxidative-induced vascular dysfunction ${ }^{(1)}$, recent studies have suggested the contribution of vascular superoxide production resulting from multi-component NADPH oxidases (NOX) within endothelial cells ${ }^{(2)}$. To this end, the present study aimed to assess the structure-activity relationships of anthocyanin derived degradation products and metabolites in the modulation of angiotensin II (Ang II) induced superoxide release from intact endothelial cells. Direct $\mathrm{Fe} /$ ion reducing capacity and inhibition of xanthine oxidase were also investigated.

Initially, several conjugated anthocyanin-derived phenolic acid metabolites ${ }^{(3)}$ were chemically synthesised and characterised by LC/MS and $\mathrm{H}^{1}$ NMR. To assess the modulation of Ang II-induced superoxide production, confluent HUVECs were exposed to physiological concentrations of treatment compounds and the reduction of cytochrome $c$ was measured at $550 \mathrm{~nm}$. Superoxide generation was determined using co-incubations in the presence of superoxide dismutase. NOX expression was confirmed by Western blot and qRT-PCR analysis. Fe/ion reducing capacity was assessed from the direct reduction of cytochrome $c$ and the inhibition of superoxide production by xanthine/xanthine oxidase was assessed spectrophotometrically following incubations in the presence of xanthine, ferricytochrome $c$ and the treatment compounds. Significance of triplicate experiments was determined (one-way ANOVA with Tukey post-hoc tests) against control or basal conditions $(P<0.05)$ and data expressed as $\%$ of controls mean (SD).

Here we present evidence that anthocyanins and their metabolites attenuate Ang II-induced endothelial superoxide production and that the cellular activity of anthocyanins was enhanced following degradation and metabolism to phenolic acid metabolites. Specifically, at concentrations of 0.1 and $1 \mu \mathrm{M}$, respectively, the glucuronide conjugate of 4-hydroxybenzoic acid [27.9 (SD 8.4) and 97.2 (SD 15.3)\% inhibition] showed a greater effect on Ang II-induced superoxide release than its precursors 4-hydroxybezoic acid (33.2 (SD 23.3 ) and 71.4 SD 16.2)\% inhibition) and pelargonidin-3-glucoside [21.0 (SD 24.7) and 22.7 (SD 18.8)\% inhibition]. Similarly, cyanidin-3-glucoside and protocatechuic acid did not appear to attenuate superoxide production at physiological concentrations, although both the glucuronide [87.9 (SD 4.1) and -1.4 (SD 18.9)\% inhibition] and methyl (0.5 (SD 17.7) and 67.6 (SD 26.3)\% inhibition] conjugates of protocatechuic acid demonstrated significant inhibitory effects.

Thus, the ability of phenolic acids to inhibit superoxide release appeared to be inversely associated with the number of aryl hydroxyls ( $\downarrow \mathrm{OH}=\uparrow$ bioactivity), which was in express opposition to their antioxidant potential ( $\uparrow \mathrm{OH}=\uparrow$ antioxidant potential), as established via Fe/ion reduction and xanthane oxidase inhibition assays. Based on this dichotomy, it is likely that the biological effects of anthocyanins on the vasculature, at physiologically relevant concentrations, are mediated by their phenolic acid degradation products and metabolites.

This study was supported in part by a BBSRC-CASE studentship (G.M.W.) in partnership with GlaxoSmithKline (UK).

1. Bedard K \& Krause KH (2007) Physiol Rev 87, 245-313.

2. Ago T, Kitazono T, Ooboshi H et al. (2004) Circulation 109, 227-233.

3. Woodward G, Needs P \& Kay C (2010) Mol Nut Food Res (Article online in advance of print); DOI 10.1002/mnfr.201000355. 